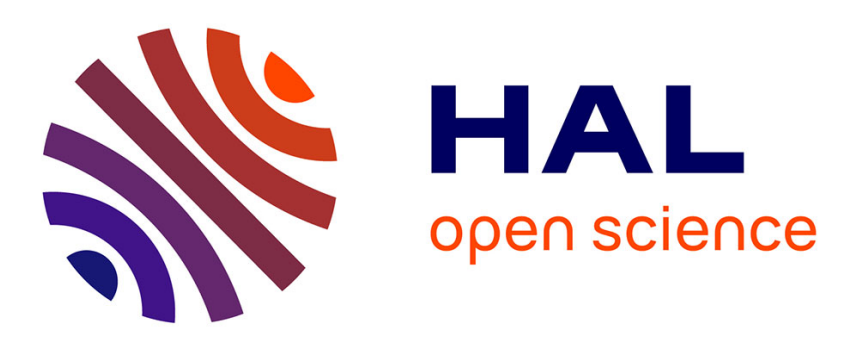

\title{
An Integrated Framework for Binary Sensor Placement and Inhabitants Location Tracking
}

Maria Pia Fanti, Gregory Faraut, Jean-Jacques Lesage, Michele Roccotelli

\section{To cite this version:}

Maria Pia Fanti, Gregory Faraut, Jean-Jacques Lesage, Michele Roccotelli. An Integrated Framework for Binary Sensor Placement and Inhabitants Location Tracking. IEEE Transactions on Systems, Man and Cybernetics, Part A: Systems and Humans, 2016, 10.1109/TSMC.2016.2597699 . hal-01367581

\section{HAL Id: hal-01367581 https://hal.science/hal-01367581}

Submitted on 16 Sep 2016

HAL is a multi-disciplinary open access archive for the deposit and dissemination of scientific research documents, whether they are published or not. The documents may come from teaching and research institutions in France or abroad, or from public or private research centers.
L'archive ouverte pluridisciplinaire HAL, est destinée au dépôt et à la diffusion de documents scientifiques de niveau recherche, publiés ou non, émanant des établissements d'enseignement et de recherche français ou étrangers, des laboratoires publics ou privés. 


\title{
An Integrated Framework for Binary Sensor Placement and Inhabitants Location Tracking
}

\author{
Maria Pia Fanti, Senior Member, IEEE, Gregory Faraut, Member, IEEE, \\ Jean-Jacques Lesage, Member, IEEE, and Michele Roccotelli
}

\begin{abstract}
This correspondence paper deals with the sensor placement optimization problem in the context of indoor multiple inhabitants location tracking to solve ambient assisted living problems. Binary sensors, like passive infrared (PIR) sensors, are used to guaranty specific coverage requirements and allow privacy respecting. Moreover, within real home environments, different kinds of obstacles (like walls, high furniture, etc.) can affect the detection capacity of PIR sensors. This paper proposes an integrated framework devoted to optimize the placement of sensors and PIR sensors in smart homes by taking into account physical topologies and coverage precision constraints. An integer linear programming problem is formalized and a case study illustrates the applicability of the proposed approach and the scalability of the optimization method.
\end{abstract}

Index Terms-Ambient assisted living (AAL), binary sensors, integer linear programming (ILP), smart home.

\section{INTRODUCTION}

Ambient assisted living (AAL) solutions are considered core issues to improve health care at home. The object is proposing innovative methods and approaches for localizing elderly people in domestic environments and identifying their daily life activities. In this context, smart home technologies allow helping people to live in comfortable and safe environments. A smart home is a flat equipped with sensors and actuator devices that can be remotely controlled. On the basis of the information given by the sensors, the actuators are controlled for different purposes, e.g., to improve the comfort (heating, air conditioning and ventilation systems, lights control, etc. [4]) or to ensure the safety of inhabitants (fire or gas control, health problem detection, etc.) [1]. The AAL research field aims at improving the comfort and the safety of aged or disabled people living in their own dwelling by including several applications such as the remote monitoring of patients having long-term diseases, the control of smart equipment to support elderly people in their daily activities, and the indoor location tracking of inhabitants [1]-[3], [12], [17], [18]. In particular, indoor location tracking is a meaningful evidence for numerous AAL activities. It consists in finding the location of inhabitants on the basis of the information given by different sensors. For instance, the knowledge of the real-time location of inhabitants is helpful for the detection

Manuscript received January 18, 2016; revised April 30, 2016; accepted July 27, 2016. This work was supported by the Italian Minister Ministero dell'Istruzione, dell'Università e della Ricerca (MIUR) in the "Smart Cities and Communities and Social Innovation" section, in the framework of project "ASMARA-Applications post Directive 2010/65 in Italian port realities of the Suite MIELE to support the Authority to optimize the inteRoperability in the intermodAlity of city-port flows" SCN_00529. This paper was recommended by Associate Editor D. Akopian.

M. P. Fanti and M. Roccotelli are with the Department of Electrical and Information Engineering, Polytechnic of Bari, 70126 Bari, Italy (e-mail: mariapia.fanti@poliba.it; michele.roccotelli@poliba.it).

G. Faraut and J.-J. Lesage are with the LURPA, ENS Cachan, University of Paris-Sud, Université Paris-Saclay, 94235 Cachan, France (e-mail: gregory.faraut@lurpa.ens-cachan.fr; jean-jacques.lesage@lurpa.ens-cachan.fr).

Color versions of one or more of the figures in this paper are available online at http://ieeexplore.ieee.org.

Digital Object Identifier 10.1109/TSMC.2016.2597699 of health problems or to improve the environment comfort [2], [3]. For these purposes, it is relevant to optimize the number and placement of sensors in smart homes in order to improve the location tracking performance [6]-[13] as well as minimizing the cost of instrumentation.

In this correspondence paper, an integrated framework devoted to provide the designers with the best solution for the binary sensor placement is proposed. The system receives in input, the data about the possible sensor choices and the apartment topology, and optimizes the placement of the binary sensors like passive infrared (PIR) sensors, by considering different aspects: 1) the obstacles, like walls, that can limit the sensors detection; 2) a topological classification of the zones in which the tracking has to be performed; and 3) coverage and precision constraints of the location. In particular, the dwelling is divided into independent zones on the basis of the presence of walls and, for each independent zone, an independent sensor placement optimization problem is formalized as an integer linear programming (ILP) problem. Moreover, the application of the presented method to a case study, including different scenarios-in terms of precision and coverage of the location-is presented and discussed.

This paper is organized as follows. Section II discusses the related works. Section III presents the structure and the components of the integrated system. Moreover, Section IV describes the phases of the optimization strategy for sensors placement and Section V shows the effectiveness of the proposed method by considering a real flat topology. Finally, Section VI summarizes the conclusions and gives some perspectives for further works.

\section{RELATED WORKS}

In the literature related to AAL problems, some studies propose several technological solutions to realize different applications mainly related to activities of daily living (ADLs) recognition and location tracking. Rashidi and Mihailidis [18] summarized the state-of-the-art of AAL technologies, tools, and techniques. They also explore successful case studies and deployed systems and discuss the present and future challenges. Magherini et al. [19] described a system that uses propositional temporal logic and model-checking to support automated real-time recognition of ADLs within a smart environment. The effectiveness of the proposed approach is shown by applying it to a smart kitchen. Moreover, Andò et al. [20] explored the possibility to detect ADLs, with a particular focus on fall detection and present a smartphone-based ADL detector, which adopts an advanced classification paradigm to distinguish between different kinds of falls. Nag and Mukhopadhyay [21] dealt with the problem of correctly recognizing the output of flexi-force sensors that may vary depending on the time usage. These sensors are typically used to monitor the occupancy or nonoccupancy status of chairs, sofa, and bed. Nag and Mukhopadhyay [21] proposed a real-time method based on dynamic thresholding that allows differentiating the two situations even in case of long-time usage. Furthermore, Zhou et al. [22] presented a case-driven ambience intelligence (C-AmI) system that 
aims to manage elderly people ADLs at home. The C-AmI system architecture integrates wireless sensor network, activity recognition, case-based reasoning, elderly in-home assistance customized knowledge within a coherent framework. It supports the sensing, predicting, and reasoning of assistive actions by exploiting the a priori knowledge about solved cases to meet the aged people's needs in a domestic environment.

Other related works in the AAL context refer specifically to the indoor inhabitants location tracking and different technologies and methodologies are used to locate the position of humans within a smart environment. Some authors develop tracking systems based on invasive sensors like wearable and/or cameras devices [23]-[27]. In this context, Tabar et al. [24] proposed a wearable identification badge, to localize a person in a flat. The user badge is equipped with two accelerometers for measuring sudden movements representing the fall of an elderly person and the approximate location of the user is computed by evaluating the signal strength measurement in the home environment. Furthermore, a distributed vision-based system for managing the accident in the assisted living environment has been developed in [25]. The posture of people is detected by merging features from different camera images in order to reduce false alarms. In [26], a wearable ear-mounted device for localizing an inhabitant in the home environment has been developed. It is composed of an infrared proximity sensor and a dual axis accelerometer. When the person walks wearing the device, the accelerometer detects the footsteps while sometimes the proximity sensor fails to recognize the doorways. The authors remark that sometimes the proximity sensor fail to recognize the doorways accurately and, as a result, the human localization is not precise.

Wearable sensor systems can localize inhabitant positions with good accuracy but several drawbacks are derived from their use. Elderly people can forget to follow the instructions and do not like to carry special devices with them and there are also privacy issues. Moreover, many of these systems require complex infrastructure to be installed at home for inhabitant localization.

Some authors deal with the localization of inhabitants by using mobile robotic systems [23], [27], [28]. Such systems are invasive and the main results of the presented methodologies are derived from simulation studies while the real life experiments are still limited.

In order to recognize the inhabitant activities in real time, by using cheap sensors and without violating the privacy, PIR motion sensors can be used. In this context, Yang et al. [29] presented a distributed human location procedure for a binary pyroelectric infrared sensor tracking system and propose a two-level regional location, with static and dynamic partitioning. Crandall and Cook [30] used multiple PIR sensors and two location tracking algorithms. Crandall and Cook [30] remarked that it is needed to install a dense grid of sensors for the full coverage of the environment but anyway in some situations the human activity is not monitored correctly by one or more sensors resulting in less accurate results.

Hence, it appears that the study of the optimal design of infrared motion sensing systems for human motion localization is an open problem. In particular, Feng et al. [6] aimed at finding the optimal number and placement of PIR sensors in order to improve the localization performance. A multiobjective, mixed-integer-discretecontinuous optimization problem is presented and solved by using a divide and conquer-based genetic algorithm method. Moreover, Emmons and Kamangar [8] evaluated the optimal placement of binary sensors by providing several definitions for sensor coverage and spatial probability and by focusing on 1-D sensor arrays. A metric for measuring the overall utility of a given set of choices is presented and includes the precision evaluation of measurement and coverage, and the associated costs. In addition, Zhao et al. [9] faced the problem of finding the optimal sensor placement that can minimize the target tracking uncertainty by studying a control strategy that provides a flexible solution to autonomous optimal sensor deployment in 2-D and 3-D spaces.

In the previously mentioned works, different methods to solve the problem of sensors placement in a given space (1-D, 2-D, or 3-D) for location tracking purposes are developed but they do not solve the problem of multiple inhabitants location tracking in smart homes by considering real environments. In this context, Gaddam et al. [12] presented a framework to care elderly people in smart home based on wireless sensors. The sensor selection architecture is performed by an evaluation module that chooses the appropriate sensor nodes on the basis of performance requirements for observability, fault detection, fault robustness, and cost. However, Gaddam et al. [12] do not show the applicability of the method on a real home environment. On the other hand, Vlasenko et al. [13] proposed a hardware/software platform that aims to help inhabitants in performing several daily home activities. The goal is to recognize the general activities in real time, by respecting the privacy and developing a method for planning the optimal deployment of binary PIR motion sensors. Vlasenko et al. [13] optimized the sensors placement on the basis of the zones visitation frequency by considering only one inhabitant. In [1]-[3], a method that aims at the systematic construction of a discrete event system (DES) model for real-time location tracking of one or multiple inhabitants into a smart home is proposed. In addition, a location tracking algorithm as well as a procedure to evaluate the relevance of a given instrumentation for location tracking, are presented. Several criteria are defined to evaluate a priori the performance of the chosen instrumentation and to help the expert for manually improving it. In particular, one of these criteria measures the inability of the sensors network to detect the presence of inhabitants in certain zones of the flat, defined as unlocationable zones. On the basis of the defined criteria, the performance of the location tracking algorithm is evaluated by an expert that can decide if a modification of sensors in terms of type, number, and placement is necessary.

In order to overcome the problem of manually modifying the sensors network by means of an expert, this paper starts from the contributions in [1]-[3] and provides an automatic procedure to optimize the placement of the sensors by guaranteeing the respect of the coverage requirements.

\section{Structure of the Integrated Framework FOR SENSOR PLACEMENT}

In this section, we describe the proposed methodology devoted to optimize the sensor placement in a generic flat topology. We present an integrated system that can be used by the designers to select the best solution for the placement of PIR sensors in order to satisfy suitable constraints and optimize a defined objective function. In particular, we are not interested in determining the identity of the inhabitants and the kinds of activities they are doing, but we aim to track the location of inhabitants in the flat with a specified accuracy. For this purpose, we are interested in optimizing the sensing system placement to reach the desired location tracking performances.

The integrated system is defined by considering the following assumptions: 1) binary sensors, like PIR sensors, are used because they are low cost, and ensure the privacy respect and 2) the living space is discretized by means of bidimensional grids because the placement of sensors and the localization of inhabitants in 2-D spaces are required for AAL purposes [11]-[14].

Moreover, the proposed integrated system structure is constituted by two main components: 1) the data component, which includes 


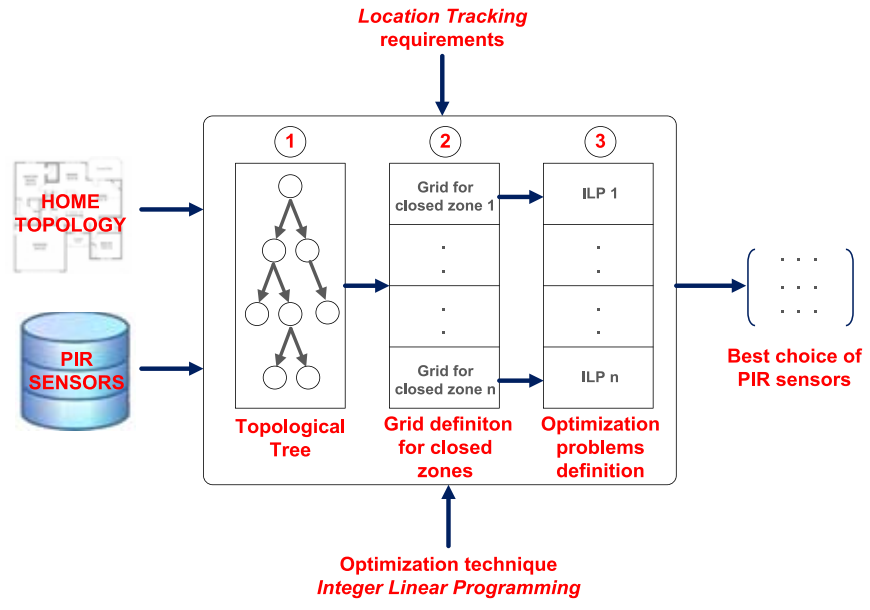

Fig. 1. Main components of the integrated framework.

a database describing the technological characteristics of the PIR sensors and the home topology map and 2) the model component, that provides the mathematical formulation of the sensor placement optimization problem by specifying the constraints and the objectives of the problem.

In particular, the model component is the core of the integrated system and is specified by the following three phase procedure.

1) Classification Phase: The zones of the dwelling that are limited by walls or obstacles (in the most cases the zones are the rooms) are classified by means of a strict order inclusion relation, which is graphically represented by a topological tree. This procedure determines a set of closed zones: we define closed zone a part of the flat where the optimization can be separately applied.

2) Discretization Phase: For each closed zone, two discretization scales are defined according to the required accuracy for sensor placement and location tracking: a) a first scale defines a grid for sensor placement and b) a second scale defines a grid for zone coverage.

3) Optimization Phase: An independent optimization problem is defined for each closed zone.

Fig. 1 gives an overview of the optimization framework structure. The main components of the system and the relationships among them are pointed out. The outputs are the solutions provided by the optimization problems that give the optimal choices of type, position, and number of the PIR sensors for each closed zone.

\section{DATA And Model COMPONEnTS OF THE INTEGRATED FRAMEWORK}

This section specifies the data and the model components of the presented system and explains in detail the proposed procedure to optimize the sensor placement.

\section{A. Data Component}

PIR sensors belong to a set of types with cardinality $S$, where each element $h \in H$ denotes a motion detector type. Moreover, each sensor type $h$ is characterized by the following attributes. A detection angle $d a_{h}$ that can be $d a_{h}=180^{\circ}$ or $d a_{h}=360^{\circ}$, a detection radius $d r_{h} \in \mathbb{N}^{+}$; a cost $c_{h} \in R^{+}$. In particular, the PIR sensors can be placed in different zones on the basis of their types. For example, sensors with $d a_{h}=180^{\circ}$ can be positioned on the walls, and sensors with $d a_{h}=360^{\circ}$ can be placed on ceilings.

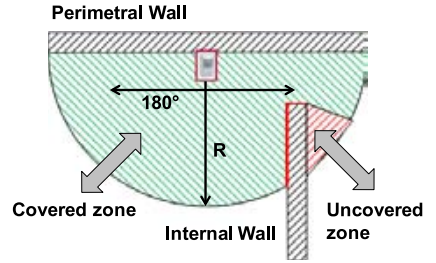

(a)

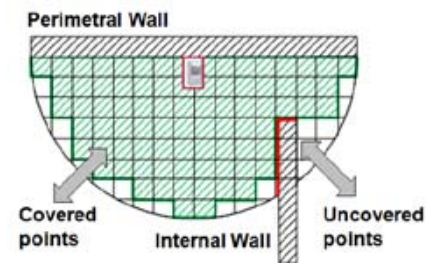

(b)
Fig. 2. Motion detectors coverage including obstacles. (a) Coverage zone. (b) Coverage points on the grid.

As an example, Fig. 2(a) shows the geometric definition of the coverage zone of a PIR sensor type with $d a_{h}=180^{\circ}$ and $d r_{h}=R$. The presence of a wall into this zone constitutes an obstacle, which generates an uncovered zone. Fig. 2(b) provides a discretization of the same coverage zone for a given coverage scale. Moreover, the closets, the doors and the windows can also be considered as obstacles for the motion detectors within the domestic environment.

\section{B. Classification Phase}

During the classification phase the topology of the flat is considered in order to define and classify the set of closed zones by means of a topological tree expressing an inclusion relationship between zones.

Here, we define as closed a zone whose space is limited by walls or materials, which constitute obstacles to the signals emitted by PIR sensors.

Each node of the tree represents a closed zone that may or not include smaller closed zones. The whole flat can be decomposed in closed zones and at the end of the decomposition the leaves of the tree have to be considered for the independent optimization application.

The procedure to build the tree is as follows.

1) The root node is the flat and is placed at the level 1, i.e., the highest level.

2) The main zones $Z c_{i}$ of the flat limited by the main walls are singled out and form the nodes of the tree second level.

3) Each zone $Z c_{i}$ is inspected in order to determine other zones $Z c_{i, j}$ limited by cupboards, furniture, partitions, etc.

4) The procedure goes to an end when no other area is highlighted.

By adopting the proposed zone classification, an independent sensor placement optimization problem can be defined for each leaf of the tree with different granularity for each level and each zone. The following example clarifies the proposed classification phase.

Example 1: Let us consider the flat shown in Fig. 3 and composed by the following rooms. An open space including the living room $(5.5 \times 5.2 \mathrm{~m})$, the kitchen and the dining room $(3.6 \times 4.2 \mathrm{~m})$; a laundry room and a pantry room; the bathroom; the master suite zone including a master suite $(4.2 \times 5.2 \mathrm{~m})$, a bathroom and a dressing room; a second bedroom $(4 \times 3.4 \mathrm{~m})$; a garage $(7 \times 6.5 \mathrm{~m})$.

Considering the topology of the flat described in Fig. 3, internal walls of the main rooms define a set of closed zones as represented in Fig. 4. Starting from level 1 (the flat) the four main closed zones belonging to level 2 are singled out: the zone $Z c_{1}$, the master suite zone $Z c_{2}$, the second bedroom $Z c_{3}$, and the garage $Z c_{4}$ (see Fig. 5).

Moreover, the following closed zones of level 3 are included in zone $Z c_{1}$ : the main open space that is represented by the node $Z c_{1.1}$; the second bathroom that is represented by the node $Z c_{1.2}$; the laundry and pantry rooms zone that is identified by the node $Z c_{1.3}$. In addition, the following closed zones of level 3 are included in zone $Z c_{3}$ : the master suite (node $Z c_{3.1}$ ); the dressing room (node $Z c_{3.2}$ ); and the bathroom (node $Z c_{3.3}$ ).

Four closed zones compose level 4: the laundry room $\left(Z c_{1.3 .1}\right)$ and the pantry room $\left(Z c_{1.3 .2}\right)$ that are included in $Z c_{1.3}$; the bath 


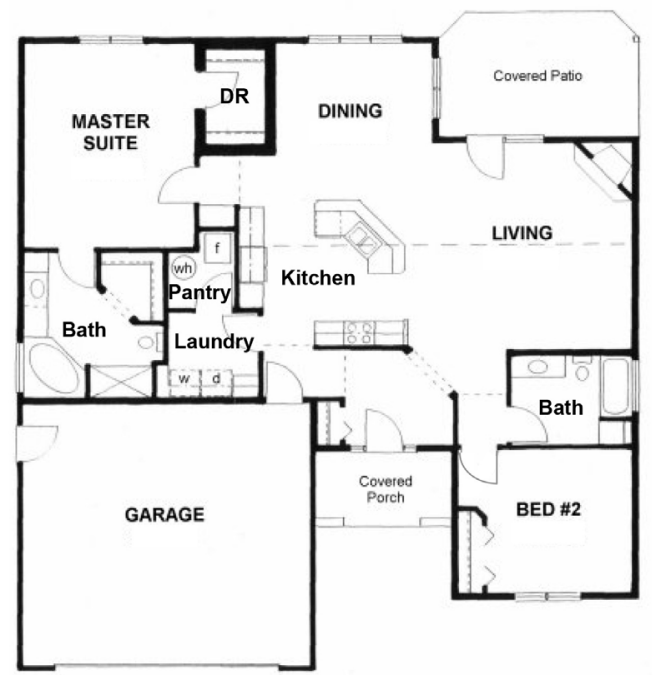

Fig. 3. Flat topology.

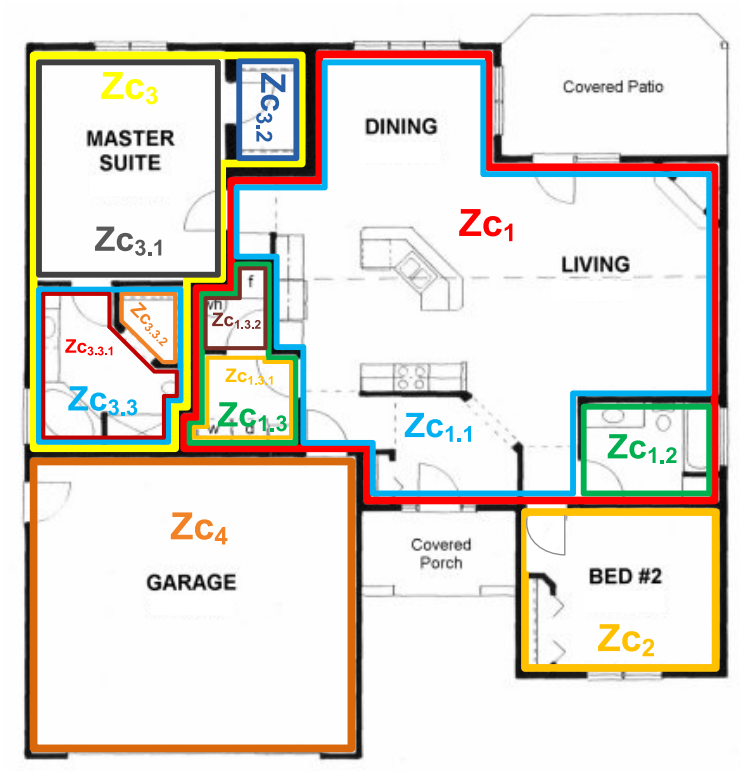

Fig. 4. Closed zones definition.

and shower (nodes $Z c_{3.3 .1}$ and $Z c_{3.3 .2}$ ) that are included in $Z c_{3.3}$. The resulting tree is shown in Fig. 5 that exhibits the leaves representing the obtained set of closed zones: $Z=\left\{Z c_{1}, Z c_{1.1}, Z c_{1.2}\right.$, $Z c_{1.3}, Z c_{1.3 .1}, Z c_{1.3 .2}, Z c_{2}, Z c_{3}, Z c_{3.1}, Z c_{3.2}, Z c_{3.3}, Z c_{3.3 .1}, Z c_{3.3 .2}$, $\left.Z c_{4}\right\}$. For each closed zone an independent optimization problem can be defined.

\section{Discretization Phase}

In order to model the optimization problem by an ILP formulation, it is necessary to discretize the flat area. Two independent discretization grid scales are defined for each zone: 1 ) the grid scale $g_{c}$ divides the zones on the basis of the user requirements in term of location accuracy and 2) the grid scale $g_{p}$ defines the granularity of the sensors placement and has to be fixed by considering the technology of the sensors, especially, the detection radius. Note that it is possible to define different grid scales, for both $g_{c}$ and $g_{p}$, for each of the 2-D: for the sake of the simplicity in the rest of this paper the same scales are considered for the 2-D.

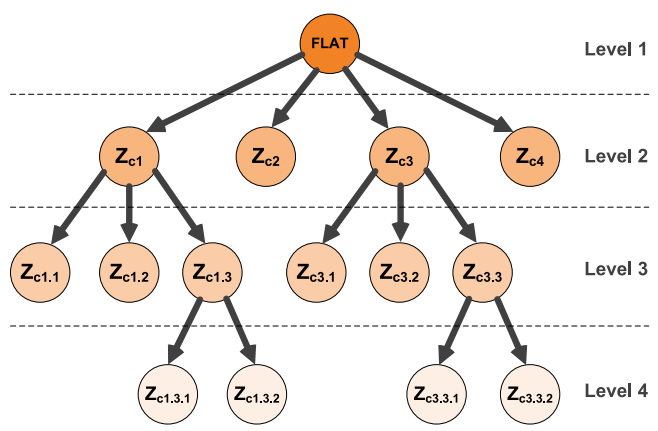

Fig. 5. Topological tree.

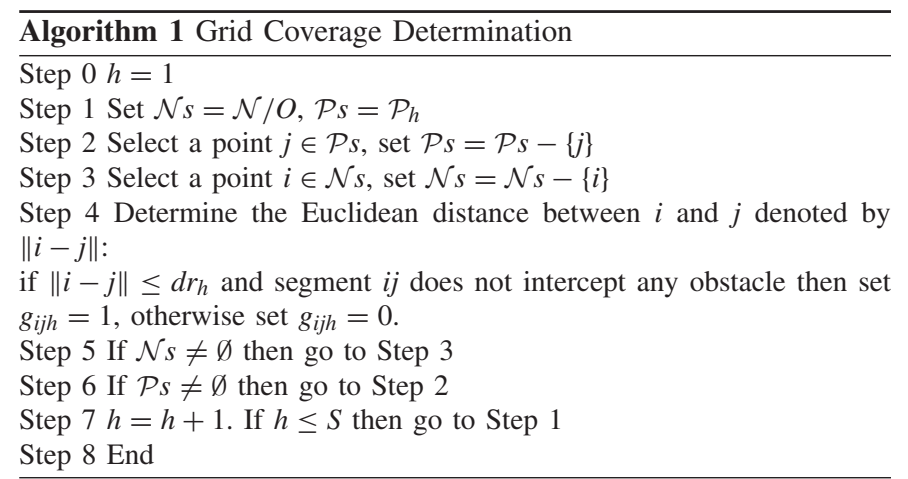

Now, in order to describe the flat discretization, the following sets are defined.

1) $\mathcal{N}=\left\{i \in \mathbb{N}^{+} \mid i=1, \ldots, N\right\}$ : Set of points obtained by discretizing the considered zone within a 2-D grid scale $g_{c}$ that is suitable for obtaining the required coverage precision.

2) $O=\{i \in \mathcal{N} \mid i$ is occupied by an obstacle $\}: O \subset \mathcal{N}$, set of points of the grid that are occupied by an obstacle.

3) $\mathcal{M}=\left\{l \in \mathbb{N}^{+} \mid l=1, \ldots, M\right\}$ : Set of points obtained by discretizing the considered zone within a 2-D grid scale $g_{p}$ that is suitable for defining the precision of sensor placement.

4) $\mathcal{P}_{h}=\left\{j \in \mathcal{M} \mid j=1, \ldots, P_{h}\right\}: \mathcal{P}_{h} \subset \mathcal{M}$, set of grid points where sensors of type $\mathrm{h}$ can be placed.

5) $\mathcal{P}=\cup_{h \in H} \mathcal{P}_{h}$ : Set of grid points (with cardinality $P$ ) where sensors can be placed.

Note that the discretization can be obtained by standard techniques [9].

\section{Optimization Problem Formulation}

In this section, we formalize the sensor placement optimization problem for each closed zone by an ILP problem. In the proposed optimization framework, the objective is to optimize the placement of the sensors, by minimizing costs and satisfying the coverage requirements.

The grid coverage is denoted by the following binary variables, given as input data for the optimization problem:

$$
g_{i j h}= \begin{cases}1, & \text { if sensor of type } h \text { that is placed } \\ & \text { at point } j \text { covers point } i \\ 0, & \text { otherwise }\end{cases}
$$

$$
\forall h \in H, j \in \mathcal{P}_{h}, i \in \mathcal{N} / O .
$$

The values of $g_{i j h}$ are determined in Algorithm 1 .

It is evident that $g_{i j h}=0, \forall i \in O$ with $h \in H$ and $j \in \mathcal{P}_{h}$.

The procedure considers for each sensor of type $h$ a possible position $j \in \mathcal{P}_{h}$ (step 2). Then, each point $i \in \mathcal{N} / O$ of the grid is selected (step 3 ) in order to evaluate the distance $\|i-j\|$. If it holds 
$\|i-j\| \leq d r_{h}$ and the segment $i j$ does not intercept any obstacle, then the considered sensor can cover point $i$ (step 4) and it holds $g_{i j h}=1$.

Moreover, the decision variables are the binary variables $x_{j h}$ defined as follows:

$$
x_{j h}= \begin{cases}1, & \text { if sensor of type } h \text { is placed at point } j \\ 0, & \text { otherwise } \\ & \forall h \in H, j \in \mathcal{P}_{h}, i \in \mathcal{N} / O .\end{cases}
$$

The goal of the problem is minimizing the costs of the sensors that have to cover the grid points $i \in \mathcal{N} / O$ according to specific requirements and by excluding the obstacles. To this purpose, we introduce the following parameters.

$a_{i} \in \mathbb{N}^{+}$if $i \in \mathcal{N} / O$, where $a_{i}$ represents the minimum number of sensors that have to cover the grid point $i$ that is not an obstacle.

$a_{i}=0$ if $i \in O$, i.e., $i$ is an obstacle.

Now, the ILP problem is formalized as follows:

$$
\begin{gathered}
\min \sum_{j=1}^{P} \sum_{h=1}^{S} c_{h} x_{j h} \\
\text { subject to } \sum_{j=1}^{P} \sum_{h=1}^{S} g_{i j h} x_{j h} \geq a_{i}, \quad \forall i \in \mathcal{N} / O .
\end{gathered}
$$

Equation (1a) is the objective function that minimizes the sum of the sensor costs by considering the grid points $j \in \mathcal{P}$ available for sensor placements. Constraints (1b) impose that the minimum number of sensors that have to cover the grid point $i \in \mathcal{N} / O$ is equal to $a_{i}$.

The ILP problem (1a), (1b) is known in the related literature as the set multicover problem that is NP-hard [32]. Even if for the dimension of the considered problems the exact solution is obtained in reasonable time, several algorithms are available in the literature and allow solving large problems [33].

\section{CAse Study}

In this section, the application of the optimization method to the flat of Example 1 and shown in Figs. 2 and 4 is discussed by considering four scenarios where different closed zones are considered. Each scenario represents a particular situation that is analyzed to show the powerful of the proposed method by considering suitable zones under specified constraints.

1) In scenario $1, Z c_{1.1}$ is considered to show the covering of a zone by properly placing the sensors in presence of permanent walls.

2) In scenario $2, Z c_{2}$ is considered to show how to cover a zone in presence of open windows and open doors that can be obstacles for the sensor detection.

3) In scenario 3, the ability of the methodology to take into account the sensor failures is studied by considering zone $Z c_{1.1}$.

In the considered case study, the database of sensors includes five types of motion detectors: $H=\{h=1, \ldots, 5\}$. Table I reports, for each sensor type the corresponding attributes: note that sensors of type $h=1,2,3$ can be positioned on the wall and sensors of type $h=4,5$ can be positioned on the ceiling. Moreover, two 2-D grids are defined for each zone and identified through their scales that are reported in Table II. A grid of scale $g_{p}$ is determined for the sensor placement and a grid of scale $g_{c}$ defines the coverage precision.

In such a way, we show that it is possible to choose the values of the grid scales on the basis of the accuracy of placement and coverage precision requirements. In the considered scenarios, the discretization of the grids is determined as follows.

Let us consider a rectangular zone $(A \times B)$ and a grid scale of size $g$. The resulting grid set $\mathcal{N}=\{i=1, \ldots, N\}$ is composed by $N=(\lfloor A / g\rfloor+1) *(\lfloor B / g\rfloor+1)$ points and each point $i$ corresponding
TABLE I

BASIC Database of PIR SENSORS

\begin{tabular}{l|ccccc}
\hline \hline & \multicolumn{5}{|c}{ Sensor type } \\
\hline $\begin{array}{l}\text { Sensor } \\
\text { attributes }\end{array}$ & Type 1 & Type 2 & Type 3 & Type 4 & Type 5 \\
$\begin{array}{l}\text { Detection } \\
\text { angle }\left(^{\circ}\right)\end{array}$ & 180 & 180 & 180 & 360 & 360 \\
$\begin{array}{l}\text { Detection } \\
\text { radius }(\mathrm{m})\end{array}$ & 4 & 8 & 12 & 6 & 10 \\
Cost $(€)$ & 35 & 50 & 60 & 40 & 50 \\
\hline \hline
\end{tabular}

\begin{tabular}{|c|c|c|c|c|}
\hline & \multicolumn{3}{|c|}{ Closed Zone / Scenario } \\
\hline & & $Z c_{1.1} / \mathrm{S} 1$ & $Z c_{2} / \mathbf{S 2}$ & $Z c_{1.1} / \mathrm{S3}$ \\
\hline \multirow{2}{*}{ Grid } & $g_{p}$ & $40 \mathrm{~cm}$ & $20 \mathrm{~cm}$ & $40 \mathrm{~cm}$ \\
\hline & $g_{c}$ & $40 \mathrm{~cm}$ & $20 \mathrm{~cm}$ & $40 \mathrm{~cm}$ \\
\hline \multicolumn{2}{|c|}{$a_{i}$} & 1 & 1 & 2 \\
\hline
\end{tabular}

TABLE II

Zones Discretization and COVERAge Constraints IN THE THREE SCENARIOS

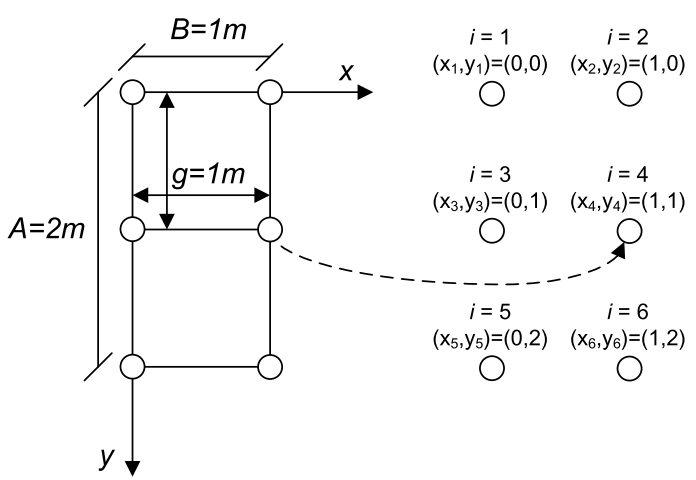

Fig. 6. Two-dimensional grid description of a simple zone $(2 \mathrm{~m} \times 1 \mathrm{~m})$.

to the grid position $\left(x_{i}, y_{i}\right)$ is determined by the following formula:

$$
i=y_{i} / g \times(\lfloor B / g\rfloor+1)+x_{i} / g+1, \text { for } i=1, \ldots, N
$$

where $\lfloor\alpha\rfloor$ denotes the floor function of $\alpha$, i.e., the largest integer not greater than the real number $\alpha$.

Example 2: Consider the rectangular zone $(A \times B)=$ $(2 \times 1) \mathrm{m}^{2}$ depicted in Fig. 6 where a grid scale of $g=1 \mathrm{~m}$ is applied. The resulting grid is composed by 6 points $\mathcal{N}=\{i=1, \ldots, 6\}$ and each point corresponds to the grid position $\left(x_{i}, y_{i}\right)$ determined according to (2) and shown in Fig. 6.

In the following sections, we solve the ILP problem (1a), (1b) by considering the three selected scenarios. The ILP problems are solved by using a standard solver, i.e., GNU linear programming kit [31] running on an Intel-Core i5-2450M, CPU at $3.40 \mathrm{GHz}$, with $6 \mathrm{~GB}$ RAM. Moreover, the solution of the problems is obtained within less than $30 \mathrm{~min}$.

\section{A. Scenario 1}

In scenario 1 , the closed zone $Z c_{1.1}$ is considered. The objective is to optimize the placement of sensors by imposing that at least one sensor covers each zone not representing obstacles, i.e., $a_{i}=1$ if $i \in \mathcal{N} / O$ and $a_{i}=0$ if $i \in O$.

Assuming that the walls and the closets are obstacles and using the grid scales reported in Table II, the zone $Z c_{1.1}$ is discretized as 


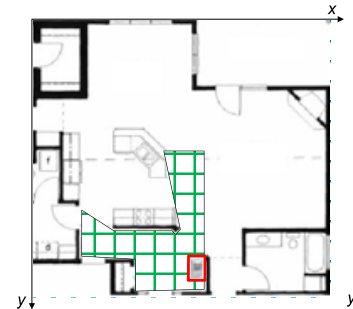

(a) (b)

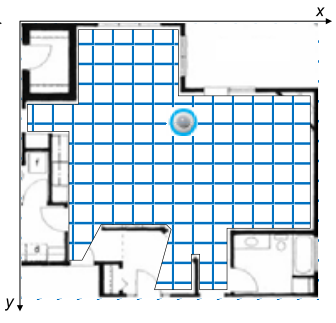

Fig. 7. ILP solution for $Z c_{1.1}$. (a) Zone covered by a sensor of type $h=1$; (b) Zone covered by a sensor of type $h=4$.

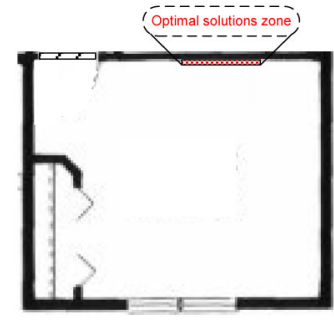

(a)

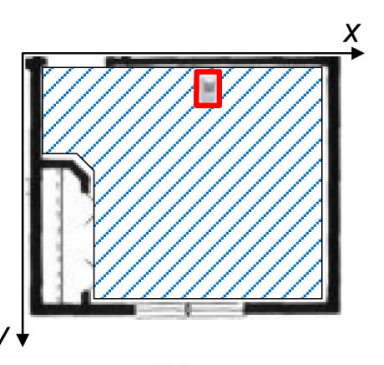

(b)
Fig. 8. ILP solutions for $Z c_{2}$ in case 2a. (a) Zone of optimal solutions. (b) First solution $\left(x_{1}, y_{1}\right)=(10,0)$, of the set $\mathcal{S}(2 a)$.

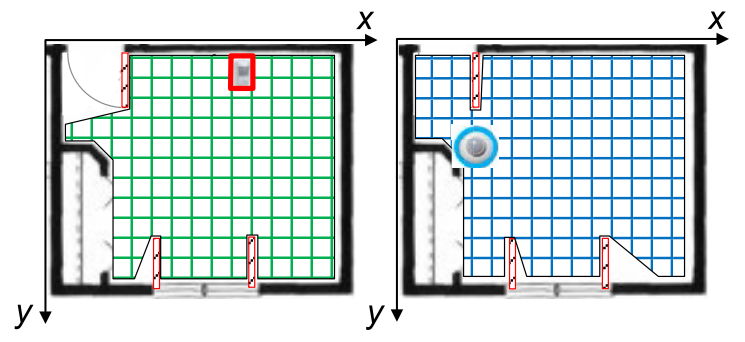

(a)

(b)

Fig. 9. ILP solution of case 2b. (a) Sensor of type $h=1$ in $\left(x_{1 a}, y_{1 a}\right)=$ $(10,0)$. (b) Sensor of type $h=4$ in $\left(x_{1 b}, y_{1 b}\right)=(6,6)$.

follows: $N=M=483, P=270$. The ILP problem has only one solution that uses two sensors of type $h=1$ and $h=4$, respectively. The two sensors are placed at the grid coordinates $\left(x_{1}, y_{1}\right)=(11,20)$ and $\left(x_{2}, y_{2}\right)=(12,6)$, respectively, as shown in Fig. 7(a) and (b).

\section{B. Scenario 2}

In scenario 2, the closed zone $Z c_{2}$ is considered and includes two cases. In case $2 \mathrm{a}$, the door and window are supposed to be closed and are no obstacles; in case $2 \mathrm{~b}$, the door and the window may be closed or opened, i.e., they have to be considered as possible obstacles. Also in this scenario, we impose that at least one sensor covers each zone not representing obstacles, i.e., $a_{i}=1$ if $i \in(\mathcal{N} / O)$ and $a_{i}=0$ if $i \in O$. The grids reported in Table $\mathrm{I}$ result in the following discretization of zone $Z c_{2}: N=M=378, P=308$.

In case $2 \mathrm{a}$, the ILP problem provides the following set of optimal solutions, composed of sensors of type $h=1$, very close together and exhibiting the same value of the objective function:

$$
\mathcal{S}(2 a)=\left\{\left(x_{s}, y_{s}\right)=(10+s, 0) \text { for } s=0, \ldots, 4\right\} .
$$

Fig. 8(a) shows the zone of the possible locations of the sensors and Fig. 8(b) shows the first solution of set $\mathcal{S}(2 a)$.

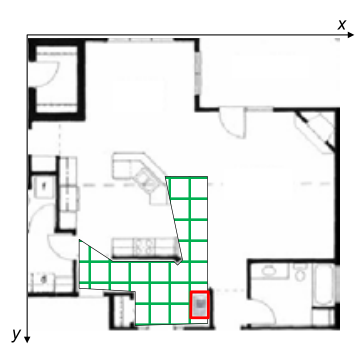

(a)

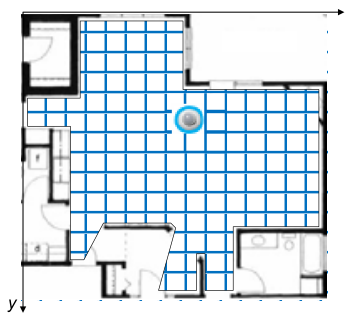

(c)

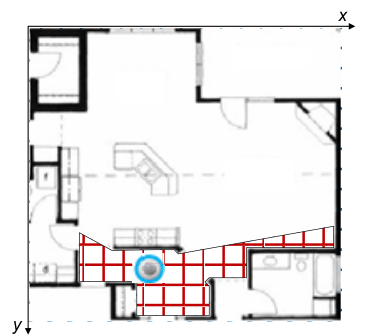

(b)

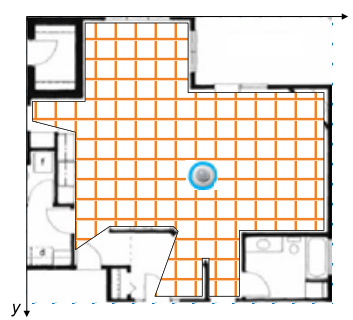

(d)
Fig. 10. ILP solution for $Z c_{1.1}$. (a) Zone covered by the sensor of type $h=1$. (b)-(d) Zone covered by the three sensors of type $h=4$.

Furthermore, the ILP problem solution of case $2 \mathrm{~b}$ provides a set of 20 optimal solutions and each solution is composed of two sensors: 1 ) one sensor of type $h=1$ and 2) one sensor of type $h=4$. The set of the solutions is the following (sensors of types 1 and 2 are placed, respectively, at $\left(x_{s a}, y_{s a}\right)$ and $\left(x_{s b}, y_{s b}\right)$, for $\left.s=1, \ldots, 20\right)$ :

$$
\begin{aligned}
& \mathcal{S}(2 b) \\
& \qquad\left\{\begin{array}{l}
\left(x_{1 a}, y_{1 a}\right)=(10,0) \text { and }\left(x_{1 b}, y_{1 b}\right)=(6,6) \\
\left(x_{2 a}, y_{2 a}\right)=(10,0) \text { and }\left(x_{2 b}, y_{2 b}\right)=(6,7) \\
\left(x_{3 a}, y_{3 a}\right)=(20,6) \text { and }\left(x_{3 b}, y_{3 b}\right)=(6,8) \\
\left(x_{4 a}, y_{4 a}\right)=(12,0) \text { and }\left(x_{4 b}, y_{4 b}\right)=(3,2) \\
\left(x_{5 a}, y_{5 a}\right)=(13,0) \text { and }\left(x_{5 b}, y_{5 b}\right)=(4,3) \\
\left(x_{6 a}, y_{6 a}\right)=(14,0) \text { and }\left(x_{6 b}, y_{6 b}\right)=(5,4) \\
\left(x_{7 a}, y_{7 a}\right)=(15,0) \text { and }\left(x_{7 b}, y_{7 b}\right)=(5,3) \\
\left(x_{8 a}, y_{8 a}\right)=(16,0) \text { and }\left(x_{8 b}, y_{8 b}\right)=(3,1) \\
\left(x_{9 a}, y_{9 a}\right)=(17,17) \text { and }\left(x_{9 b}, y_{9 b}\right)=(3,3) \\
\left(x_{10 a}, y_{10 a}\right)=(20,10) \text { and }\left(x_{10 b}, y_{10 b}\right)=(3,4) \\
\left(x_{11 a}, y_{11 a}\right)=(20,3) \text { and }\left(x_{11 b}, y_{11 b}\right)=(3,5) \\
\left(x_{12 a}, y_{12 a}\right)=(20,15) \text { and }\left(x_{12 b}, y_{12 b}\right)=(4,4) \\
\left(x_{13 a}, y_{13 a}\right)=(20,14) \text { and }\left(x_{13 b}, y_{13 b}\right)=(4,5) \\
\left(x_{14 a}, y_{14 a}\right)=(20,12) \text { and }\left(x_{14 a}, y_{14 a}\right)=(5,7) \\
\left(x_{15 a}, y_{15 a}\right)=(20,7) \text { and }\left(x_{15 b}, y_{15 b}\right)=(3,6) \\
\left(x_{16 a}, y_{16 a}\right)=(16,17) \text { and }\left(x_{16 b}, y_{16 b}\right)=(4,7) \\
\left(x_{17 a}, y_{17 a}\right)=(20,13) \text { and }\left(x_{17 b}, y_{17 b}\right)=(4,6) \\
\left(x_{18 a}, y_{18 a}\right)=(20,8) \text { and }\left(x_{18 b}, y_{18 b}\right)=(5,5) \\
\left(x_{19 a}, y_{19 a}\right)=(20,9) \text { and }\left(x_{19 b}, y_{19 b}\right)=(5,6) \\
\left(x_{20 a}, y_{20 a}\right)=(20,1) \text { and }\left(x_{20 b}, y_{20 b}\right)=(4,2)
\end{array}\right\} .
\end{aligned}
$$

In particular, Fig. 9 shows the first solution of set $\mathcal{S}(2 b)$.

\section{Scenario 3}

Scenario 3 considers the closed zone $Z c_{1.1}$ and imposes that at least two sensors cover each grid point $i$ not belonging to an obstacle, i.e., $a_{i}=2$ if $i \in \mathcal{N} / O$ and $a_{i}=0$ if $i \in O$. Using the grids shown in Table I, the zone is discretized as follows: $N=M=483, P=270$.

The ILP solution returns only one optimal solution that includes four sensors: one sensor of type $h=1$ is placed at the grid coordinates $(x, y)=(11,20)$ and three sensors of type $h=4$ are placed at the grid coordinates $(x, y)=(7,16),(x, y)=(13,7)$, and $(x, y)=$ $(13,12)$, respectively. 
The placements of the sensors are shown in Fig. 10. In this case, it is possible to perform a location tracking even in case of a sensor failure.

\section{CONCLUSiON}

This correspondence paper presents an integrated framework to optimize the sensor placement in a multiple inhabitant locationtracking by using binary sensors. The integrated system is composed of a database describing the flat and the sensors and a model component that provides the optimal sensor placements.

To this aim, the flat is divided into a set of independent closed zones and the proposed method optimizes the placement of sensors within each zone by minimizing the sensor costs. The proposed method allows an accurate placement of sensors and a complete coverage of each zone by discretizing the space in bidimensional grids with different scales and by considering the problem of obstacles for motion detectors. On the basis of a real smart home topology, three different optimization scenarios are presented and discussed to demonstrate the effectiveness of the optimization method that may provide different alternative optimal solutions that the user can choose. The solutions presented in the considered case study show the benefit of the proposed method: it is possible to cover the flat with the minimum number of sensors by guaranteeing full coverage and addressing sensor failures.

Future research will consider other kinds of sensors in order to improve the accuracy of the coverage and the performance of the location tracking.

\section{REFERENCES}

[1] M. Danancher, J.-J. Lesage, L. Litz, and G. Faraut, "A discrete event model for multiple inhabitants location tracking," in Proc. IEEE Int. Conf. Autom. Sci. Eng. (CASE), Madison, WI, USA, Aug. 2013, pp. $910-915$.

[2] M. Danancher, J.-J. Lesage, L. Litz, and G. Faraut, "Online location tracking of a single inhabitant based on a state estimator," in Proc. IEEE Int. Conf. Syst. Man Cybern. (SMC), Manchester, U.K., Oct. 2013, pp. 391-396.

[3] M. Danancher, J.-J. Lesage, and L. Litz, "Model-based location tracking of an a priori unknown number of inhabitants in smart homes," IEEE Trans. Autom. Sci. Eng., vol. 13, no. 2, pp. 1090-1101, Apr. 2016, doi: 10.1109/TASE.2015.2477558.

[4] M. P. Fanti, A. M. Mangini, M. Roccotelli, and W. Ukovich, "A district energy management based on thermal comfort satisfaction and realtime power balancing," IEEE Trans. Autom. Sci. Eng., vol. 12, no. 4, pp. 1271-1284, Oct. 2015.

[5] N. K. Suryadevara, C.-P. Chen, S. C. Mukhopadhyay, and R. K. Rayudu, "Ambient assisted living framework for elderly wellness determination through wireless sensor scalar data," in Proc. 7th Int. Conf. Sens. Technol. (ICST), Wellington, New Zealand, Dec. 2013, pp. 632-639.

[6] G. Feng, Y. Yang, X. Guo, and G. Wang, "Optimal design of infrared motion sensing system using divide-and-conquer based genetic algorithm," in Proc. IEEE Int. Conf. Mechatron. Autom. (ICMA), Takamatsu, Japan, Aug. 2013, pp. 482-487.

[7] A. N. Bishop, B. Fidan, B. D. O. Anderson, K. Doğançay, and P. N. Pathirana, "Optimality analysis of sensor-target localization geometries," Automatica, vol. 46, no. 3, pp. 479-492, Mar. 2010.

[8] S. P. Emmons and F. Kamangar, "Evaluating the optimal placement of binary sensors," Int. J. Inf. Sci. Tech., vol. 3, no. 1, pp. 1-10, Jan. 2013.

[9] S. Zhao, B. M. Chen, and T. H. Lee, "Optimal deployment of mobile sensors for target tracking in 2D and 3D spaces," IEEE/CAA J. Autom. Sinica, vol. 1, no. 1, pp. 24-30, Jan. 2014.

[10] K. Farkas, "Placement optimization of reference sensors for indoor tracking," Acta Polytech. Hungarica, vol. 12, no. 2, pp. 123-139, 2015.

[11] A. Hiromori, H. Yamaguchi, and T. Higashino, "Sensor placement optimization method for people tracking," in Proc. 7th Int. Conf. Next Gener. Mobile Apps Services Technol. (NGMAST), Prague, Czech Republic, Sep. 2013, pp. 62-67.
[12] A. Gaddam, K. Kaur, S. C. Mukhopadhyay, and G. S. Gupta, "Selection and optimization of wireless sensors in a smart digital home for the elderly," in Proc. IEEE Sensors, Christchurch, New Zealand, Oct. 2009, pp. 1382-1386.

[13] I. Vlasenko, I. Nikolaidis, and E. Stroulia, "The smart-condo: Optimizing sensor placement for indoor localization," IEEE Trans. Syst., Man, Cybern., Syst., vol. 45, no. 3, pp. 436-453, Mar. 2015.

[14] M. Younis and K. Akkaya, "Strategies and techniques for node placement in wireless sensor networks: A survey," Ad Hoc Netw., vol. 6, no. 4, pp. 621-655, Jun. 2008.

[15] Y. Zou and K. Chakrabarty, "Uncertainty-aware and coverage-oriented deployment for sensor networks," J. Parallel Distrib. Comput., vol. 64, no. 7, pp. 788-798, Jul. 2004.

[16] S. S. Dhillon, K. Chakrabarty, and S. S. Iyengar, "Sensor placement for grid coverage under imprecise detections," in Proc. 5th Int. Conf. Inf. Fusion, vol. 2. Annapolis, MD, USA, Jul. 2002, pp. 1581-1587.

[17] T. Kovácsházy and G. Fodor, "New approach to passive infrared motion sensors signal processing for ambient assisted living applications," in Proc. IEEE Int. Conf. Instrum. Meas. Technol. (I2MTC), Graz, Austria, May 2012, pp. 2521-2525.

[18] P. Rashidi and A. Mihailidis, "A survey on ambient-assisted living tools for older adults," IEEE J. Biomed. Health Inform., vol. 17, no. 3, pp. 579-590, May 2013.

[19] T. Magherini, A. Fantechi, C. D. Nugent, and E. Vicario, "Using temporal logic and model checking in automated recognition of human activities for ambient-assisted living," IEEE Trans. Human-Mach. Syst., vol. 43, no. 6, pp. 509-521, Nov. 2013.

[20] B. Andò, S. Baglio, C. O. Lombardo, and V. Marletta, "An event polarized paradigm for ADL detection in AAL context," IEEE Trans. Instrum. Meas., vol. 64, no. 7, pp. 1814-1825, Jul. 2015.

[21] A. Nag and S. C. Mukhopadhyay, "Occupancy detection at smart home using real-time dynamic thresholding of flexiforce sensor," IEEE Sensors J., vol. 15, no. 8, pp. 4457-4463, Aug. 2015.

[22] F. Zhou, J. R. Jiao, S. Chen, and D. Zhang "A case-driven ambient intelligence system for elderly in-home assistance applications," IEEE Trans. Syst., Man, Cybern. C, Appl. Rev., vol. 41, no. 2, pp. 179-189, Mar. 2011.

[23] S. A. Mehdi and K. Berns, "A survey of human location estimation in a home environment," in Proc. 23rd IEEE Int. Symp. Robot Human Interact. Commun., Edinburgh, U.K., Aug. 2014, pp. 135-140.

[24] A. M. Tabar, A. Keshavarz, and H. Aghajan, "Smart home care network using sensor fusion and distributed vision-based reasoning" in Proc. 4th ACM Int. Workshop Video Surveillance Sensor Netw., Santa Barbara, CA, USA, 2006, pp. 145-154.

[25] H. Aghajan, J. C. Augusto, C. Wu, P. McCullagh, and J.-A. Walkden, "Distributed vision-based accident management for assisted living," in Pervasive Computing for Quality of Life Enhancement. Heidelberg, Germany: Springer-Verlag, 2007, pp. 196-205.

[26] G. Schindler, C. Metzger, and T. Starner, "A wearable interface for topological mapping and localization in indoor environment," in Locationand Context-Awareness. Heidelberg, Germany: Springer, 2006.

[27] J. Koch, J. Wettach, E. Bloch, and K. Berns, "Indoor localisation of humans, objects, and mobile robots with RFID infrastructure," in Proc. 7th Int. Conf. Hybrid Intell. Syst. (HIS), Kaiserslautern, Germany, Sep. 2007, pp. 271-276.

[28] M. Volkhardt, S. Mueller, C. Schroeter, and H.-M. Gross, "Playing hide and seek with a mobile companion robot," in Proc. 11th IEEE-RAS Int. Conf. Humanoid Robots (Humanoids), Bled, Slovenia, Oct. 2011, pp. 40-46.

[29] B. Yang, Y. Lei, and B. Yan, "Distributed multi-human location algorithm using naive bayes classifier for a binary pyroelectric infrared sensor tracking system," IEEE Sensors J., vol. 16, no. 1, pp. 216-223, Jan. 2016.

[30] A. S. Crandall and D. J. Cook. "Tracking systems for multiple smart home residents," in Proc. Human Behav. Recognit. Technol. Intell. Appl. Monitor. Security, 2011, pp. 111-129.

[31] GLPK (GNU Linear Programming Kit), Free Softw. Found. Inc., Boston, MA, USA, 2012.

[32] C. Chekuri, K. L. Clarkson, and S. Har-Peled, "On the set multicover problem in geometric settings," ACM Trans. Algorithms, vol. 9, no. 1, p. 9, 2012.

[33] Q.-S. Hua, D. Yu, F. C. M. Lau, and Y. Wang, "Exact algorithms for set multicover and multiset multicover problems," in Algorithms and Computation. Heidelberg, Germany: Springer, 2009, pp. 34-44. 